\title{
BMJ Open Protocol for a scoping review to evaluate the extent of utilisation of healthcare services by asthma patients in sub- Saharan African countries
}

\author{
Pisirai Ndarukwa (D) , ${ }^{1,2}$ Moses John Chimbari, ${ }^{3}$ Elopy Sibanda ${ }^{4}$
}

To cite: Ndarukwa $P$, Chimbari MJ, Sibanda E.

Protocol for a scoping review to evaluate the extent of utilisation of healthcare services by asthma patients in sub-Saharan African countries. BMJ Open 2021;11:e046294. doi:10.1136/ bmjopen-2020-046294

- Prepublication history and additional supplemental material for this paper are available online. To view these files, please visit the journal online. (http://dx.doi.org/10.1136/ bmjopen-2020-046294).

Received 26 0ctober 2020 Accepted 29 July 2021

\section{Check for updates}

(C) Author(s) (or their employer(s)) 2021. Re-use permitted under CC BY-NC. No commercial re-use. See rights and permissions. Published by BMJ.

${ }^{1}$ Public Health, University of KwaZulu-Natal, Durban, South Africa

${ }^{2}$ Department of Health Sciences, BIndura University of Science Education, Bindura, Zimbabwe ${ }^{3}$ School of Nursing and Public Health, University of KwaZuluNatal, Durban, South Africa ${ }^{4}$ Medical School, National University of Science and Technology Faculty of Medicine, Bulawayo, Zimbabwe

Correspondence to Dr Pisirai Ndarukwa; papandarukwa@gmail.com

\section{ABSTRACT}

Introduction Health systems in sub-Saharan African (SSA) countries are fragile and centralised. Consequently, majority of people have restricted access to healthcare services. Given the rise in the prevalence and burden of asthma in SSA, it is imperative to scrutinise the utilisation of healthcare services by people with asthma. We aim to understand, through this review, the extent of utilisation of healthcare services by asthma patients in SSA countries. Methods and analysis Arksey and O'Malley's scoping review methodology framework will be used to guide the conduct of this scoping review. We will conduct a search of the literature on the electronic databases: Medline, (using PubMed interface), EMBASE, EBSCOHOST, Web of Science and Google Scholar, grey literature sources and the reference lists of key studies to identify studies appropriate for inclusion. Two reviewers will independently screen all abstracts and full-text studies for inclusion. Registration of the proposed scoping review on the PROSPERO has indicated that no similar work has been or is being done elsewhere. We will review studies published on the subject from January 2009 to May 2020 in SSA.

Ethics and dissemination The proposed scoping review will contribute towards the knowledge base on utilisation of healthcare services particularly for people with asthma. This will provide a better understanding of the extent of utilisation of healthcare services by asthma patients and ultimately contribute to improvement of quality of care for people suffering from asthma. The results from the review will enlighten and guide healthcare practitioners and researchers on developing appropriate and feasible interventions to increase the utilisation of healthcare services by asthma patients in resource-constrained settings in SSA countries. Results of this scoping review will be disseminated through a peer-reviewed publication, conference presentations and a 1-day stakeholder meeting.

PROSPERO registration number CRD42020154127.

\section{BACKGROUND}

Healthcare utilisation is the quantification or explanation of the use of services by persons for the purpose of preventing and curing health problems, promoting maintenance of health and well-being, or obtaining information about one's health status and
Strengths and limitations of this study

- This will be the first scoping review to assess the utilisation of healthcare services by asthma patients in sub-Saharan African countries.

- The search strategy includes four electronic databases with peer-reviewed literature, including article bibliographies and numerous conference proceedings, as well as a broad range of grey literature sources, including government and other organisation websites including healthcare utilisation by asthma.

- Preferred Reporting Items for Systematic reviews and Meta-Analyses extension for Scoping Reviews guidelines will be followed for this scoping review.

- Although, Andersen's behavioural model has been used extensively in studies investigating the use of health services it has not addressed healthcare services utilisation for asthma patients. Though this study will evaluate the extent of healthcare utilisation of services by asthma patients, there will be no formal interviews with patients or even healthcare providers.

- A major limitation will be the inclusion of only English language studies.

prognosis. ${ }^{12}$ Health status and the need for healthcare services to improve or maintain health are major determinants of healthcare utilisation. ${ }^{3}$ This study will thus evaluate the extent of utilisation of healthcare services by patients with asthma in the ub-Saharan African countries (SSA).

Asthma is defined as a heterogeneous chronic respiratory disorder characterised by the presence of intermittent symptoms such as wheezing, coughing, chest tightness and shortness of breath that are typically associated with reversible airflow obstruction and resolve spontaneously or after treatment. ${ }^{4}$ It accounts for a significant global morbidity and healthcare costs. ${ }^{5}$ Approximately, 235 million people suffer from asthma and a mortality of over 300000 reported annually. ${ }^{6}$ 
Over the last 40 years, the global rate of asthma has increased by $50 \%$ every decade and has contributed to severe disability in 19.4 million people. ${ }^{7}$ The prevalence of asthma varies between countries, ranging between $1 \%$ and $18 \% .^{8}$ In the SSA, the prevalence of asthma is less studied or understood. ${ }^{9}$ The prevalence in highly developed countries including the UK, USA and Canada generally ranges between $10 \%$ and $20 \% .^{10}$ Although its frequency has been rising, especially in urban areas, asthma is less common in developing countries, as would be expected given the role of industrial air pollutants in triggering this condition. ${ }^{11}$ Other reasons why there are differences in the asthma proportions in high-income countries compared with low-income to middle-income countries (LMICs) could be best explained by the hygiene hypothesis. ${ }^{12}$ The hygiene hypothesis suggests that increase in rates of asthma worldwide may be due to reduced exposure to bacteria and viruses. ${ }^{13}$

Asthma presents major challenges to households and health systems. ${ }^{14}$ These challenges include the challenges in diagnoses, challenges in treatment, follow-up challenges and other general challenges. ${ }^{15}{ }^{16}$ In the LMIC, it contributes to disability in nearly 16 million people and is often associated with negative effects on the quality of life. ${ }^{17}$ Asthma mainly affects economically disadvantaged people in developed nations while in the developing nations it is more common among the affluent population owing to increased cleanliness and smaller family sizes in the developed nations and among the affluent society of the LMICs. ${ }^{18}$ WHO estimates that about a quarter of a million people, most in LMICs die of asthma annually. ${ }^{19}$ In other words, because of limited access to medical treatment for asthma, rates of affliction may be lower but rates of mortality higher in less developed nations. ${ }^{20}$ Thus, asthma patients who admitted to hospitals with acute symptoms are disproportionately from economically disadvantaged groups. ${ }^{20}$

Given the increased prevalence of asthma in SSA, it is imperative to scrutinise the utilisation of healthcare services by people affected by asthma using the Anderson Health Behaviour Model. The use of the Anderson Health Behaviour Model will improve the explanation of the concepts likely to influence the extent of utilisation of healthcare services that include age, gender, education, ethnicity and beliefs. Further, the model can assist the reviewers to be able to assess measures of access (eg, equitable, inequitable, effective, efficient) as well as understand the environment (eternal or healthcare system) impacting on access nd utilisation of healthcare services. Health systems (HS) in SSA countries are fragile and centralised resulting in the majority of people having restricted access to the healthcare services. ${ }^{21}$ Healthcare services are generally much better in urban areas and households of high socioeconomic status in Africa. ${ }^{22}$ Factors including distant from health facilities, cultural barriers to healthcare access, ${ }^{23}$ the cost of care and poor awareness and appreciation of personal health may affect healthcare-seeking practices for asthma. ${ }^{24}$
Governments in SSA have committed to improving health outcomes through numerous programmes, including the Sustainable Development Goals (SDGs), where the third goal (SDG 3) aims to ensure, among other things, universal health coverage and a one-third reduction of premature mortality from non-communicable diseases through prevention and treatment by 2030 .

Ndarukwa et $a l^{24}$ showed that knowledge of asthma among healthcare workers and patients determine the level of utilisation of healthcare services. This is in agreement with the findings by Rosas-Salazar $e t a l^{25}$ who showed that poor health literacy is a barrier to utilisation of healthcare services for asthma treatment.

In the proposed scoping review, we aim to use the Andersen Health behaviour model, ${ }^{26}$ which is widely accepted as a reliable tool for health services utilisation. According to Anderson model, healthcare service utilisation is a sequential and conditional function of three sets of factors: predisposing (demographic and social) factors, enabling (economic) factors and need (health outcomes) factors. Predisposing factors reflect the individuals' propensity to use healthcare services, enabling factors are the resources that may facilitate access to services, and the need factors represent potential needs of health services use, such as self-perceived health, chronic conditions (asthma) and restricted activity. ${ }^{27}$

\section{RATIONALE}

To our knowledge, there has been no comprehensive review of the extent to healthcare utilisation of by asthma patients in SSA countries. Case fatality rate of asthma in SSA is high among asthma patients. ${ }^{28} 29$ Therefore, study of the extent of utilisation of healthcare services by asthma patients will assist to unpack those factors that may be contributing to high disease burden in SSA. To appropriately tailor utilisation of healthcare services by asthma patients, the research community must first identify relevant factors that could impact utilisation of healthcare services that have been and may be effective at reaching these target populations as well as identify gaps in the literature that indicate further investigation. For example, the outer setting may be highly relevant in utilisation of healthcare services by asthma patients. At the same time, the review may reveal the possible outcomes for healthcare services utilisation by asthma patients (eg, policy changes).

\section{AIM}

The aim of this scoping review is to evaluate the extent of the utilisation of healthcare services by asthma patients in SSA countries.

\section{OBJECTIVES}

To describe the predisposing factors to the utilisation of healthcare services by asthma patients in SSA countries. 
To establish the enabling factors which influence the utilisation of healthcare services by asthma patients in SSA countries.

To identify the possible health outcomes for utilisation of healthcare services by asthma patients in SSA countries.

\section{METHODS}

In the proposed scoping review, we will use the Preferred Reporting Items for Systematic Reviews and MetaAnalyses for Scoping Reviews (PRISMA) checklist (see online supplemental file). ${ }^{30}$ The review will make use of the scoping review design informed by a scoping review framework which was developed by Arksey and O'Malley. ${ }^{31}$ The scoping design will be arranged in this protocol according to Arksey and O'Malley's ${ }^{31}$ six stages, which we expand on below. According to this methodology, there are five different stages in undertaking a scoping review: (1) identifying the research question (RQs); (2) identifying potentially relevant studies; (3) selecting eligible studies; (4) charting the data; (5) collating, summarising and reporting the results. We anticipate that the results of our scoping review will contribute towards a better appreciation of healthcare utilisation by asthma patients in SSA countries, thus removing potential barriers to healthcare utilisation of services by asthma patients.

\section{PATIENT AND PUBLIC INVOLVEMENT}

A review on healthcare utilisation of services by asthma patients is essential if we are to avert potential patient mortality and improving their survival. Therefore, this review will eventually benefit the body of knowledge related to healthcare utilisation of services. However, patients have not been involved in the design nor the conduct of this study. As this concerns a review, this study has no participants.

\section{STAGE 1: IDENTIFYING THE RQ}

This first stage will involve identification of clearly articulated (1) broad RQ that acts as a roadmap for the subsequent stages and (2) specific questions. We will carry out this scoping review to answer this broad question and the proceeding specific RQs:

To what extent do asthma patients utilize the healthcare services in Sub Saharan African countries?

The specific study questions that this scoping review intends to address are:

1. What are the predisposing factors to the utilisation of healthcare services by asthma patients in SSA countries?

2. How can the enabling factors, influence the utilisation of healthcare services by asthma patients in SSA countries?

3. What are possible health outcomes for utilisation of healthcare services by asthma patients in SSA countries?

\section{STAGE 2: IDENTIFICATION OF RELEVANT STUDIES}

This stage involves balancing the extensiveness and depth of the review with feasibility.

\section{SEARCH METHODS}

We will search for literature on major electronic databases (ie, Medline, (using the PubMed interface), EMBASE, EBSCOHOST and Web of Science. The secondary source of potentially relevant material will be a search of the grey or difficult to locate literature, including Open Grey, Open Access Theses and Dissertations, Google scholar, the websites of WHO and other organisations. We will perform handsearching of the reference lists of included studies, relevant reviews or other relevant documents. We will use a comprehensive search strategy that uses search terms that relate to our key concepts and that combines search terms within a concept with the Boolean terms 'OR,' combines search terms between concepts with the Boolean term 'AND,' and is adapted to the syntax used by each database. The experts (PN and MJC) will collaborate to develop the search strategy. We will the do the piloting search all the alternative words in the search strategy. We use the basic search terms which are as follows;-

((("Asthma"[Mesh] OR Asthmas OR Bronchial Asthma OR Asthma, Bronchial) AND ("Healthcare utilization"[Mesh] OR Utilization OR Utilisation OR Determinants OR Health services OR Access to care OR Hospitalization OR Inpatient care OR Outpatient Care OR Admission OR Length of stay OR LOS) ) AND (Africa filter: Angola OR Benin OR Botswana OR Burkina Faso OR Burundi OR Cameroon OR Cape Verde OR Central African Republic OR Chad OR Comoros OR Congo OR Democratic Republic of Congo OR Djibouti OR Equatorial Guinea OR Eritrea OR Ethiopia OR Gabon OR Gambia OR Ghana OR Guinea OR Guinea Bissau OR Ivory Coast OR Cote d'Ivoire OR Kenya OR Lesotho OR Liberia OR Madagascar OR Malawi OR Mali OR Mauritania OR Mauritius OR Mozambique OR Namibia OR Niger OR Nigeria OR Principe OR Reunion OR Rwanda OR Sao Tome OR Senegal OR Seychelles OR Sierra Leone OR Somalia OR South Africa OR Sudan OR Swaziland OR Tanzania OR Togo OR Uganda OR Western Sahara OR Zambia OR Zimbabwe OR Central African OR West Africa OR Western African OR Eastern African OR South African OR Southern Africa OR sub Saharan Africa))

\section{LIMITED TO 2009 IN ENGLISH AND ON HUMAN STUDIES}

The Boolean operators with exhaustive combinations of search terms for asthma condition such as Asthma, bronchial Asthma, bronchial, cardiac asthma and occupational asthma; and terms for indicators of utilisation of healthcare services; healthcare utilisation, admission, inpatient care, outpatient care, hospitalisation and access to care. Following the removal of duplicate papers, articles not considering asthma healthcare-seeking behaviour and treatment will be excluded based on title and abstract 

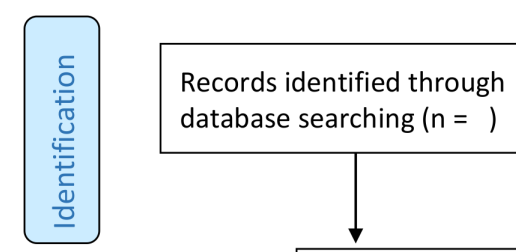

Additional records identified

through other sources $(\mathrm{n}=)$

Eventually those sources must be spelt out

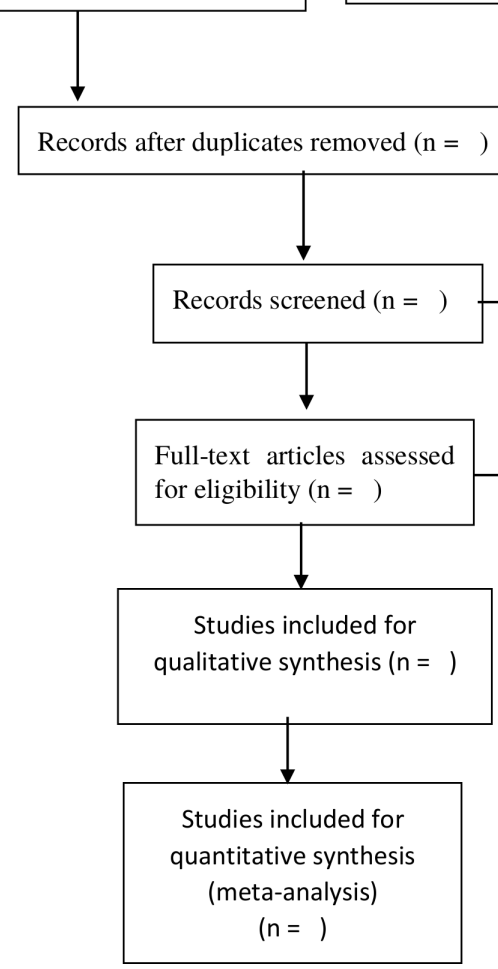

Figure 1 PRISMA 2009 flow diagram. PRISMA, Preferred Reporting Items for Systematic Reviews and Meta-Analyses.

screening. Reference lists of selected papers will be checked to identify additional studies for inclusion in the study. We will follow the PRISMA guidelines (figure 1).

\section{INCLUSION/EXCLUSION CRITERIA}

We will include published research articles in English focusing on healthcare utilisation of services by asthma patients in SSA countries. This review will exclude articles that are unavailable as full texts, and due to feasibility related reasons (eg, limited resources, including funding to hire translators), those that are not published in English. Studies not addressing utilisation of healthcare services by patients with asthma will also be excluded in this review.

\section{STAGE 3: STUDY SELECTION}

The proposed scoping review will consider all articles pertaining to utilisation of asthma healthcare services in the SSA countries. The scoping review will consider both quantitative and qualitative study designs. Studies included in the scoping review will be those reporting on utilisation of asthma healthcare services. They should be analytic and or descriptive in nature. Studies published from January 2009 to May 2020 will be included to reflect utilisation of healthcare services for diagnosis and treatment of asthma among both adults and children. These studies from January 2009 to May 2020 will be selected because they are going to offer a more robust and current state of utilisation of healthcare services by patients with asthma. The studies from January to May 2020 will ensure that the literature reflects the current health utilisation context and the nature of this dynamic process. A quick search of the data bases has also shown that there are few studies before the year 2009 focusing on the extent of healthcare services utilisation by asthmatic patients. Only studies with abstracts published in English will be included in the initial screening process.

\section{STUDY SELECTION: SCREENING}

Prior to commencing the screening process, a calibration exercise will be conducted to ensure reliability in correctly selecting articles for inclusion. It will entail independently screening a random sample of $5 \%$ of the included citations by two reviewers (PN and $\mathrm{MJC}$ ). Eligibility criteria will be modified if low agreement is observed between the reviewers (eg, a kappa statistic less than 50\%). The reviewers will then independently screen the remainder of the search results using a predefined relevance criteria form for all levels of screening (for example, title and abstract, full-text review). Discrepancies will be resolved by discussion with a third reviewer (ES). 


\begin{tabular}{ll}
$\begin{array}{l}\text { Table } 1 \text { Variables that we propose to extract, by } \\
\text { overarching }\end{array}$ \\
\hline Category & Variable \\
\hline $\begin{array}{l}\text { Characteristics of the } \\
\text { study authors }\end{array}$ & $\begin{array}{l}\text { Surnames } \\
\text { Countries (that authors' primary } \\
\text { institution are located in) } \\
\text { Lens/disciplinary points of view }\end{array}$ \\
Study methodology & $\begin{array}{l}\text { Study design } \\
\text { Sample size } \\
\text { Study population }\end{array}$ \\
Characteristics of & $\begin{array}{l}\text { Study name } \\
\text { Publication }\end{array}$ \\
Findings from the study & $\begin{array}{l}\text { Journal. } \\
\text { Research findings for } \\
\text { predisposing factors, enabling } \\
\text { factors and health outcomes/ } \\
\text { need. }\end{array}$ \\
\hline
\end{tabular}

\section{STAGE 4: CHARTING (EXTRACTING) THE DATA}

A template for the data extraction process has been created (see table 1). The extraction will focus on the study details, methods, objectives and the key findings related to the scoping review questions. Relevant data, including the study title, year of publication, study population, type of publication, study methods, geographical focus, thematic focus and key findings, will be extracted from each publication included on an excel sheet.

For each included article, we initially propose to extract (1) descriptions of elements that underlie utilisation of healthcare services for patients with asthma, as well as (2) and proposed relations between these elements. These data directly correspond to our stated RQs. In addition, we propose to extract general descriptive data specific to the study authors, publication and model/framework to provide context and to support a richer interpretation of our findings of elements that overlap and diverge with the model of healthcare utilisation. Reviewers (PN and $\mathrm{MJC}$ ) will meet with the other author (ES) twice during the process and after they extract data from the studies. At these meetings, authors will discuss any need to modify the data extraction form and document rationale for any proposed modifications. They will only make changes to the data extraction form after consultation with the entire research team.

\section{STAGE 5: COLLATING, SUMMARISING AND REPORTING THE RESULTS}

This stage involves considerations related to data analysis, reporting of results and implications of findings. We will provide a descriptive analysis of the characteristics (ie, elements that underlie healthcare utilisation of services by patients with asthma, study authors, publication and model/framework) of included articles, as well as a narrative analysis of the data. Our primary objective is to count and describe elements of healthcare utilisation of services by patients with asthma. Initial presentation of extracted data will be to the research team, and elements will be labelled using language found in the original articles. Through consultation with the research team, we may collapse elements into over-arching elements. We envision that these findings will have implications for research and practice, as well as healthcare policy strengthening. For example, this scoping review will make the readers more aware of how to improve healthcare utilisation of services by patients with asthma. It will also identify commonalities in how the Andersen's health behaviour model conceptualises healthcare utilisation of services by patients with asthma. This will serve as a solid starting point for understanding of what influences patients' healthcare utilisation of services. That said, we acknowledge that patients with asthma healthcare utilisation of services does not represent a 'one size fits all' approach.

\section{STAGE 6: CONSULTATION}

This stage is optional in Arksey and O'Malley's original framework for conduction scoping reviews, whereas Levac et al recommend that this stage is an essential component of scoping review methodology. However, in our study, we will not reach this stage as we only focus on reviewing those studies that focuses on healthcare utilisation of services by asthma patients based on Anderson model. There will be no direct consultation of patients.

\section{QUALITY APPRAISAL}

Quality of each included study will be appraised. All the selected articles will be judged for their quality based on Joanna Briggs Institute (JBI) quality appraisal tool. ${ }^{32} \mathrm{JBI}$ quality appraisal tools will be used to determine whether the articles are of sufficient quality or not. ${ }^{33} \mathrm{~A}$ careful assessment of risk of bias in the included studies will be performed by two reviewers (PN and MJC), who will first independently assess the quality of each study against each criterion. Results will be shared and agreed, and any disagreements will be addressed through engaging a third reviewer (ES). These tools were developed primarily for use in reviews. Insufficient quality of articles refers to articles whose methods for data collection, analysis and findings are not congruent. The results will help to determine if studies are consistent with the stand quality appraisal for articles.

\section{DATA SYNTHESIS}

Andersen's behavioural model will be used to categorise characteristics and determinants for healthcare utilisation of services by patients with asthma into predisposing, enabling and need characteristics. Andersen's model assumes that healthcare utilisation is a function of (1) characteristics that predispose people to use or not to use services, although such characteristics are not directly responsible for use (eg, age, gender, education, ethnicity and beliefs); (2) enabling characteristics that 
facilitate or impede use of services (income/wealth/ insurance as ability to pay for services, expenditure, organisation of service provision and health policy) and (3) needs or conditions that laypeople or healthcare providers recognise as requiring medical treatment. The model also distinguishes between individual and contextual (measured at aggregate level, such as measures of community characteristics) determinants of healthcare service utilisation. Andersen hypothesised that the variables would have differential ability to explain care use, depending on the type of service. For example, asthma care (and other discretionary services) would be explained by predisposing and enabling characteristics, whereas hospital care would primarily be explained by needs and demographic characteristics. ${ }^{345}$

Authors will extant all data according to five general classifications, including study characteristics, predisposing characteristics, enabling characteristics; need characteristics and expenditure categories and healthcare utilisation. A narrative summary will be presented under the following themes: study details, key findings and gaps in the research. The team will work together to identify key knowledge gaps emerging from the review on the main questions. All studies will be analysed according to payer and country to identify the most significant drivers across backgrounds.

\section{ETHICS AND DISSEMINATION}

The proposed scoping review is aimed at contributing to the knowledge base on healthcare utilisation of services by patients with asthma. This is considered an important step to improving care for patients with asthma and for informing interventional research related studies on asthma. Furthermore, the findings will inform programmes aimed at improving quality of care for asthma patients. We will use our findings to advocate for better financing of healthcare facilities to improve utilisation of healthcare services by patients with asthma. Furthermore, our results may guide healthcare practitioners, policy-makers and researchers on developing appropriate and feasible interventions and policies to increase utilisation of healthcare services by patients with asthma in resource-constrained settings in SSA countries.

The reviewers did not seek ethical approval for conducting the review as it will not involve access to individual-level data. The study has been registered with the PROSPERO. Findings from this review will be communicated to a broad range of stakeholders. We will also publish the review paper in an accredited journal. To ensure uptake of the review results, reviewers will also engage with policy-makers and research funders.

\section{DISCUSSION}

The results of this study will establish what is currently known about the extent of healthcare services utilisation by asthma patients in the SSA countries. To our knowledge, this scoping review protocol is the first on this topic and will identify key gaps that needs to be explored to improve healthcare services utilisation by asthma patients. The review will inform our subsequent primary research and contribute useful insights for the utilisation of the healthcare services by asthma patients.

The main limitation concerns the time filter (studies published between 2009 and 2020 will be included in this scoping review). However, this will enable us to make an updated, direct comparison of the findings with the available evidence at the District Health Information Systems on the extent of utilisation of healthcare services. Moreover, as the process of scoping reviews does not include critical quality assessment and appraisal of the studies included, the findings reported may lack reliability and validity.

Acknowledgements The authors would like to thank the UKZN and OAK foundation who have supported the reviewers throughout the process of this review.

Contributors PN and MJC conceived the review idea. PN with the assistance of MJC designed the search strategy and revisions were done by ES. PN designed the protocol. PN wrote the first draft of the manuscript and all authors (PN, MJC and ES) reviewed changes. All authors read and approved the manuscript for submission to the journal.

Funding PN is a Research Fellow with the UKZN and OAK Foundation. However, OAK Foundation and UKZN College of Health Sciences have no role in finding the study.

Competing interests None declared.

Patient consent for publication Not required.

Provenance and peer review Not commissioned; externally peer reviewed.

Supplemental material This content has been supplied by the author(s). It has not been vetted by BMJ Publishing Group Limited (BMJ) and may not have been peer-reviewed. Any opinions or recommendations discussed are solely those of the author(s) and are not endorsed by BMJ. BMJ disclaims all liability and responsibility arising from any reliance placed on the content. Where the content includes any translated material, BMJ does not warrant the accuracy and reliability of the translations (including but not limited to local regulations, clinical guidelines, terminology, drug names and drug dosages), and is not responsible for any error and/or omissions arising from translation and adaptation or otherwise.

Open access This is an open access article distributed in accordance with the Creative Commons Attribution Non Commercial (CC BY-NC 4.0) license, which permits others to distribute, remix, adapt, build upon this work non-commercially, and license their derivative works on different terms, provided the original work is properly cited, appropriate credit is given, any changes made indicated, and the use is non-commercial. See: http://creativecommons.org/licenses/by-nc/4.0/.

\section{ORCID iD}

Pisirai Ndarukwa http://orcid.org/0000-0002-6815-8088

\section{REFERENCES}

1 Carrasquillo O. Health Care Utilization, in Encyclopedia of Behaviora Medicine. In: Gellman MD, Turner JR, eds. Springer New York: New York, NY, 2013: 909-10.

2 Aday LA, Andersen RM. Health care utilization and behavior, models of. Encyclopedia of Biostatistics 2005;15.

3 National Academies of Sciences, E. and Medicine. Health-Care utilization as a proxy in disability determination. National Academies Press, 2018.

4 Becker AB, Abrams EM. Asthma guidelines: the global initiative for asthma in relation to national guidelines. 2017;17:99-103.

5 Ndarukwa P, Chimbari MJ, Sibanda EN. Development of a framework for increasing asthma awareness in Chitungwiza, Zimbabwe. Asthma Res Pract 2019;5:4. 
6 Adeloye D, Chan KY, Rudan I, et al. An estimate of asthma prevalence in Africa: a systematic analysis. Croat Med J 2013;54:519-31.

7 Network, G.A. The global asthma report 2018, 2018.

8 Nunes C, Pereira AM, Morais-Almeida M. Asthma costs and social impact. Asthma Res Pract 2017;3:1

9 Obaseki DO, Awoniyi FO, Awopeju OF, et al. Low prevalence of asthma in sub Saharan Africa: a cross sectional community survey in a suburban Nigerian town. Respir Med 2014;108:1581-8.

10 Ellwood P, Asher MI, Billo NE, et al. The global asthma network rationale and methods for phase I global surveillance: prevalence, severity, management and risk factors. Eur Respir J 2017;49:1601605.

11 Jiang X-Q, Mei X-D, Feng D. Air pollution and chronic airway diseases: what should people know and do? J Thorac Dis 2016;8:E31.

12 Stiemsma L, Reynolds L, Turvey S, et al. The hygiene hypothesis: current perspectives and future therapies. ITT 2015;4:143.

13 Bloomfield SF, Stanwell-Smith R, Crevel RW, Pickup J. Too clean, or not too clean: the hygiene hypothesis and home hygiene. Clinical \& Experimental Allergy. 2006 Apr;36(4):402-25.

14 Onyedum C, Ukwaja K, Desalu O, et al. Challenges in the management of bronchial asthma among adults in Nigeria: a systematic review. Ann Med Health Sci Res 2013;3:324-9.

15 Partridge MR. Asthma: 1987-2007. what have we achieved and what are the persisting challenges? Prim Care Respir J 2007;16:145-8.

16 O'Byrne PM. Global guidelines for asthma management: summary of the current status and future challenges. Pol Arch Med Wewn 2010;120:511-7.

17 WHO. World report on disability: World Health organization. WHO Press, 2011. https://www.who.int/disabilities/world report/2011/ report.pdf

18 Nunes C, Pereira AM, Morais-Almeida M. Asthma costs and social impact. Asthma research and practice. 2017 Dec;3(1):1-1..

19 WHO. Fact-sheet on Asthma [Electronic resource] 2019.

20 Jie Y, Isa ZM, Jie X, et al. Urban vs. rural factors that affect adult asthma. Rev Environ Contam Toxicol 2013;226:33-63.

21 Adugna MB, Nabbouh F, Shehata S, Ghahari S. Barriers and facilitators to healthcare access for children with disabilities in low and middle income sub-Saharan African countries: a scoping review. BMC health services research. 2020 Dec;20(1):1-1.

22 Kevany S, Murima O, Singh B, et al. Socio-Economic status and health care utilization in rural Zimbabwe: findings from project accept (HPTN 043). J Public Health Africa 2012;3:13-51.

23 Almutairi KM. Culture and language differences as a barrier to provision of quality care by the health workforce in Saudi Arabia. Saudi Med J 2015;36:425-31.

24 Ndarukwa P, Chimbari MJ, Sibanda EN. Protocol on a systematic review of qualitative studies on asthma treatment challenges experienced in sub-Saharan Africa. Syst Rev 2019;8:149.

25 Rosas-Salazar C, Apter AJ, Canino G, et al. Health literacy and asthma. J Allergy Clin Immunol 2012;129:935-42.

26 Andersen R. A behavioral model of families' use of health services, 1968: 25.

27 Jang Y, Chiriboga DA, Allen JY, et al. Willingness of older KoreanAmerican adults to use hospice. J Am Geriatr Soc 2010;58:352-6.

28 Stanciole AE, Ortegón M, Chisholm D, et al. Cost effectiveness of strategies to combat chronic obstructive pulmonary disease and asthma in sub-Saharan Africa and South East Asia: mathematica modelling study. BMJ 2012;344:e608.

29 Musafiri S, Joos G, Van Meerbeeck JP. Asthma, atopy and COPD in sub-Saharan countries: the challenges. East Afr J Public Health 2011;8:161-3.

30 Tricco AC, Lillie E, Zarin W, et al. PRISMA extension for scoping reviews (PRISMA-ScR): checklist and explanation. Ann Intern Med 2018;169:467-73.

31 Arksey H, O'Malley L. Scoping studies: towards a methodological framework. Int J Soc Res Methodol 2005;8:19-32.

32 Munn Z, Moola S, Riitano D, et al. The development of a critical appraisal tool for use in systematic reviews addressing questions of prevalence. Int J Health Policy Manag 2014;3:123-8.

33 Munn Z, Tufanaru C, Aromataris E. JBI's systematic reviews: data extraction and synthesis. Am J Nurs 2014:114:49-54.

34 Andersen RM, behavior s. Revisiting the behavioral model and access to medical care: does it matter? J Health Soc Behav 1995;36:1-10.

35 Kominski GF. Changing the US health care system: key issues in health services policy and management. John Wiley \& Sons, 2013. 